\title{
Within-day replanning of exceptional events
}

\section{Working Paper}

Author(s):

Dobler, Christoph; Kowald, Matthias; Schüssler, Nadine; Axhausen, Kay W. (D)

Publication date:

2011-07

Permanent link:

https://doi.org/10.3929/ethz-a-006686351

\section{Rights / license:}

In Copyright - Non-Commercial Use Permitted

\section{Originally published in:}

Arbeitsberichte Verkehrs- und Raumplanung 700 


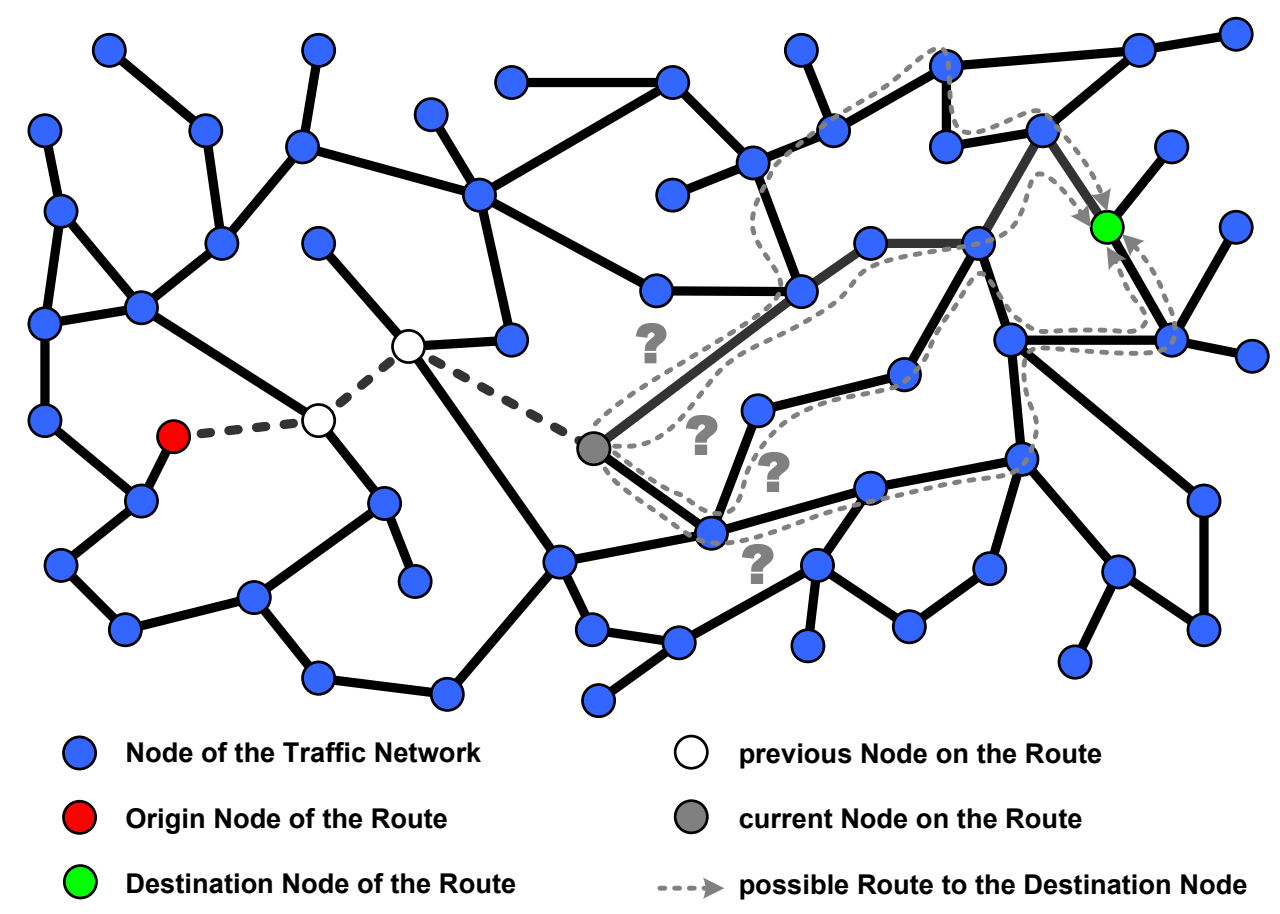

\section{Within-Day Replanning of Exceptional Events}

Christoph Dobler

Matthias Kowald

Nadine Schüssler

Kay W. Axhausen
Working paper

Transport and Spatial Planning

\footnotetext{
$\square \square \sqrt{\text { Institut für Verkehrsplanung und Transportsysteme }}$
}

\section{EH}

Eidgenössische Technische Hochschule Zürich Swiss Federal Institute of Technology Zurich 


\section{Within-Day Replanning of Exceptional Events}

\author{
Christoph Dobler \\ IVT \\ ETH Zurich \\ CH-8093 Zurich \\ phone: +41-44-633 6529 \\ fax: +41-44-633 1057 \\ dobler@ivt.baug.ethz.ch \\ Kay W. Axhausen \\ IVT \\ ETH Zurich \\ CH-8093 Zurich \\ phone: +41-44-633 3943 \\ fax: +41-44-633 1057 \\ axhausen@ivt.baug.ethz.ch
}

July 2011

\author{
Matthias Kowald \\ IVT \\ ETH Zurich \\ CH-8093 Zurich \\ phone: +41-44-633 3089 \\ fax: +41-44-633 1057 \\ kowald@ivt.baug.ethz.ch
}

\author{
Nadine Schüssler \\ IVT \\ ETH Zurich \\ CH-8093 Zurich \\ phone: +41-44-633 3085 \\ fax: +41-44-633 1057 \\ schuessler@ivt.baug.ethz.ch
}

\section{Abstract}

Typical software that is used for the simulation of traffic behavior focuses on scenarios that describe common situations like an ordinary working day without remarkable incidents. To simulate such scenarios, iterative approaches are used. They assume that the simulated people adapt to the results of the previous iterations. Such iterative approaches produce meaningful results for various scenarios as long as only typical, repetitive situations are modeled.

However, a scenario may also contain incidents that occur by chance, which increases the complexity of a model substantially. In such scenarios, an iterative approach would produce illogical and even totally wrong results. An attempt to handle such scenarios is within-day replanning.

This paper describes the problems that arise when applying an iterative simulation approach to a scenario with exceptional events. The within-day replanning technique is introduced and implemented in the multi-agent transport simulation framework MATSim. The implementation allows the simulated agents to replan the routes between their activities while they are traveling. By doing this, they can take the current traffic load into account, an important requirement for scenarios containing unpredictable incidents like road accidents.

The capability of the implementation is demonstrated by conducting experiments with a scenario where the capacities of several arterial roads in the center of the City of Zurich are reduced due to an exceptional event. It is shown, that the agents that are affected by those events are able to reduce their travel times if they replan their routes using within-day replanning. 


\section{Keywords}

Within-Day Replanning, En-Route Replanning, Re-Routing, MATSim

\section{Preferred citation style}

Dobler, C., Kowald, M., Schüssler, N. and Axhausen, K. W. (2011) Within-Day Replanning of Exceptional Events, Working paper, 700, Institute for Transport Planning and Systems (IVT), ETH Zurich, Zurich. 


\section{Introduction}

Due to events like the devastating tsunami that hit coastal regions around the Indian Ocean in December 2004, the devastating earthquake of the coast of Japan in March 2011 as well as the subsequent tsunami or the terrorist attacks on September 11th, the interest in the topic of large scale evacuation simulations has grown enormously. In the field of transport planning and traffic management, this leads to the necessity to simulate scenarios where exceptional events occur that cannot be foreseen. This requirement is in conflict with traditional simulation approaches that optimize the traffic demand using an iterative approach. There, it is assumed that a typical situation is simulated and therefore the agents can rely on their experience from comparable situations, like previous iterations.

Applying an iterative approach to a scenario with unexpected events results in problems such as illogical behavior of the agents and consequently wrong results. In the next section these problems as well as an alternative simulation approach are presented. On the one hand, this approach—called within-day replanning — simulates only a single iteration and therefore avoids the problems resulting from an iterative simulation process. On the other hand, it requires a more detailed behavioral model for the agents. Subsequently, MATSim, a framework for iterative, agent-based traffic flow micro-simulations, is described. Based on that software, the iterative approach is discussed. Afterwards, the implementation of the within-day replanning approach into the MATSim framework is presented, which includes a discussion of the technical implementation as well as the behavioral model. Then the capability of the extended version of the MATSim framework is demonstrated using a model of the city of Zurich. Multiple simulation runs are performed using different parameter settings for the behavioral model. The paper closes with some conclusions and an outlook on further work.

\section{Problem Definition}

\subsection{Exceptional Events}

Basically, an exceptional events is an incident that cannot (e.g. an earthquake) or only partially (e.g. a major sports events where the number of sold tickets is known but the impact on the transport system can only be estimated) be foreseen. Thereby, not every kind of exceptional event has the same influence on the behavior of the population. On the one hand, the number of affected people can vary, on the other hand the reaction of those people will depend on the kind of event. A small-scale incident like an accident on a minor road, will affect only few people. Probably, most of them will just wait until the traffic jam dissolves or try to find 
an alternative route. In general, such an incident will not encourage them to alter their daily schedule significantly. In contrast, large-scale events, like natural or man-made disasters, that affect the majority of the population will often lead to major changes in the daily schedule of the affected people.

Our long term intention is to develop a tool to model and simulate arbitrary exceptional events. We will focus on large-scale evacuation scenarios because they can affect large parts of the population as well as the network structure which results in very high requirements for the simulation framework.

Many studies have been conducted in the field of traffic flow simulation of large-scale evacuations. One major problem when modeling evacuations is that common traffic flow simulation frameworks are not designed to support exceptional events. Their iterative approach leads to an illogical behavior of the population and, therefore, to wrong results. To overcome these problems, studies conducted so far have either used models that have been developed with a special focus on evacuations or existing simulation frameworks that have been extended with additional features. Some examples for the first group are MASSVAC (Hobeika and Kim, 1998), MITSIMLab (Jha et al., 2004) and NETVACl (Sheffi et al., 1982). Some of them have been developed for specific kinds of disasters which cause an evacuation, e.g. NETVACl for scenarios with incidents in nuclear power plants or MASSVAC for the simulation of evacuations triggered by a hurricane. The second group of evacuation studies that is based on existing traffic flow simulation frameworks extends a simulation frameworks and/or adapts the representation of the simulated area, e.g. by changing the capacities of affected network structures. Examples are Cova and Johnson (2003), who use PARAMICS (Cameron and Duncan, 1996) and Balakrishna et al. (2008) who utilize DynaMIT (Ben-Akiva et al., 1998).

However, both groups mentioned above suffer from the problem that they still cannot model exceptional events adequately. Therefore, we introduce the within-day replanning approach, which is based on performing only a single iteration. There, the population optimizes its plans not from iteration to iteration but within this single iteration, which means that the agents are able to adapt their daily plans in order to react to an exceptional event.

\subsection{Behavior Under Evacuation Conditions}

According to our current objective of modeling and simulating large-scale evacuations, we determine the requirements for the behavioral model by looking at people's behavior under evacuation conditions. Surveying information on evacuations and formulating a behavioral model is done in a three-step approach. First, a literature review comparing people's behavior in different large-scale evacuations has been performed with the aim to get familiar with evacuations 
and related behavioral patterns. As part of the future work, the findings from this review will be validated in expert interviews and a nationally representative study for Switzerland (Kowald et al. 2011), before they will finally be used to formulate and implement a behavioral model into the within-day replanning framework presented in this paper.

Large comparative studies on people's evacuation behavior were chosen as the starting point (see Zelinsky and Kosiński, 1991; Quarantelli, 1980; Perry, 1985). Wherever possible, additional studies were used to investigate a topic in depth. In order to avoid bias from smaller evacuation events like emergencies in single households or buildings, exclusively evacuation areas above three kilometers (1.9 miles) radius were taken into consideration.

Our main findings suggest that evacuees tend to behave in a rather rational way. Although panic does occur it is the exception and mostly limited to few individuals. Panic requires specific conditions: A strong thread towards the individual existence in combination with vanishing opportunities to escape whereby time is a crucial factor (Fritz and Marks, 1954; Drabek, 1969, Rosengren et al., 1975; Houts et al., 1984). The more time available, the larger is the influence of social norms and, consequently, the more pro-social evacuees' behavior (Frey et al., 2011).

Whenever possible, nuclear families evacuate as units. If separated at the time the evacuation order is announced they rather prefer to meet within the evacuation zone than leaving individually (Fritz and Marks, 1954; Drabek, 1969; Kiefer et al., 2009). In terms of socio-demographics, women and children are more likely to participate in an evacuation than men (Houts et al., 1984; Johnson and Zeigler, 1986; Houts et al., 1988; Efrat, 1992). Furthermore, younger people participate more often than older ones (Houts et al., 1988; Liverman and Wilson, 1981; Fussell, 2006). There seems to be no clear relation between evacuation behavior and socio-economical status. On the one hand, people with a higher status are more likely to participate, on the other hand, people from a lower status level do often live in threatened areas, e.g. next to factories, plants, rail tracks etc, for which they participate in evacuations frequently (Houts et al., 1984, Efrat, 1992, Liverman and Wilson, 1981; Fussell, 2006; (Shenhar et al., 2008).

If possible, evacuees use their own car to leave a threatened area and try to find shelter close to the evacuation border. Their distribution behind this border follows a distance decay function (Liverman and Wilson, 1981; Houts et al., 1988; Zeigler et al., 1965).

Various types of influence result form people's social networks. First, people prefer homes of kin or friends to find shelter rather than going to public shelters (Houts et al., 1988; Liverman and Wilson, 1981; Shenhar et al., 2008). Second, social contacts play a crucial roll in the process of receiving and interpreting information on the evacuation. Naturally, many people are reluctaned to leaving their homes. A broad mass of people does not begin evacuation until an official order is announced. Therefore, it is important that this initial warning reaches as many people as possible, which requires daytime-specific strategies (Stern and Sinuany-Stern, 1989). 
Subsequently, most recipients of the initial warning start spreading the news in their social network. Their behavior is less motivated by the wish of warning others than rather finding confirmation for the message they received. The more the evacuation order is confirmed and taken seriously, the more likely becomes the participation of those looking for confirmation (Quarantelli, 1980; Houts et al., 1984; Quarantelli, 1990). Other, the participation increasing effects, result from the way the message is received. Face-to-face contacts have a larger impact than electronically spread messages. Messages received or confirmed by officials, such as firefighters, policemen or other institutions are taken more seriously than warnings via mass media. In addition, it is of high importance that all messages correspond to each other and contradictory information are avoided (Drabek, 1969; Houts et al., 1988).

\section{Simulation Approaches}

\subsection{Iterative Simulation Approaches}

The starting point of an iterative simulation approach, as it is used in agent-based traffic flow micro-simulations like DYNEMO (Schwerdtfeger, 1984), MATSim (Balmer, 2007) or TRANSIMS (Smith et al. . 1995), is to generate an initial plan for each agent (e.g. based on census and / or travel diary data). A plan contains an agent's intended schedule of activities and the trips that connect them. For each activity, its type (e.g. work, leisure or shopping), its location and the expected start and end time are given. A trip is specified by a transport mode and a route. The iterative optimization process consists of three steps. First, the plans are executed by a mobility simulation. Then they are evaluated by using a fitness function. Finally, the agents have to select the plans which will be executed in the next iteration. Each agent can keep several plans in its memory. Bad plans can be deleted, new plans can be created by cloning and adapting existing ones using information (e.g. travel times) from one or multiple previous iterations. The allowed adaption operations define the search space of the optimization (e.g. routes, location and start / end times of activities). This iterative optimization can be seen as a period-to-period replanning strategy. Since one day is a very commonly used duration, it is often denoted as day-to-day replanning strategy.

An iterative day-to-day replanning approach is appropriate as long as the scenario describes a typical situation or day. For such scenarios it is feasible to assume, that the agents are familiar with the typically occurring events like traffic jams in the peak hours. Therefore, they can try to avoid driving during those times or use alternative routes with less traffic. However, if the scenario contains unexpected events that the agents cannot foresee (e.g. accidents or heavy weather conditions), using an iterative approach is not the best choice. First, a user equilibrium 
will not be reached in such a scenario because the agents do not have enough information to choose optimal routes and daily activity plans. Another problem is the optimization process itself. Even if an agent chooses its routes absolutely randomly due to a lack of information, it will eventually find a good route if it tries enough different routes.

Figure 1: Exceptional event in a network

(a) Network with planned route

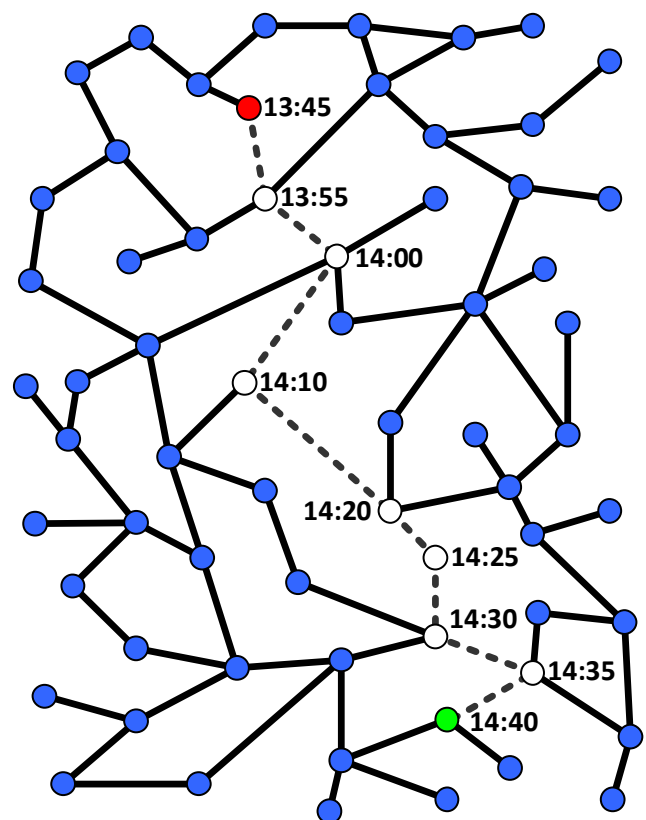

Startnode of the route $\bigcirc$ Node on the planned route Endnode of the route -.-- Planned route

(c) Network with exceptional event and planned route

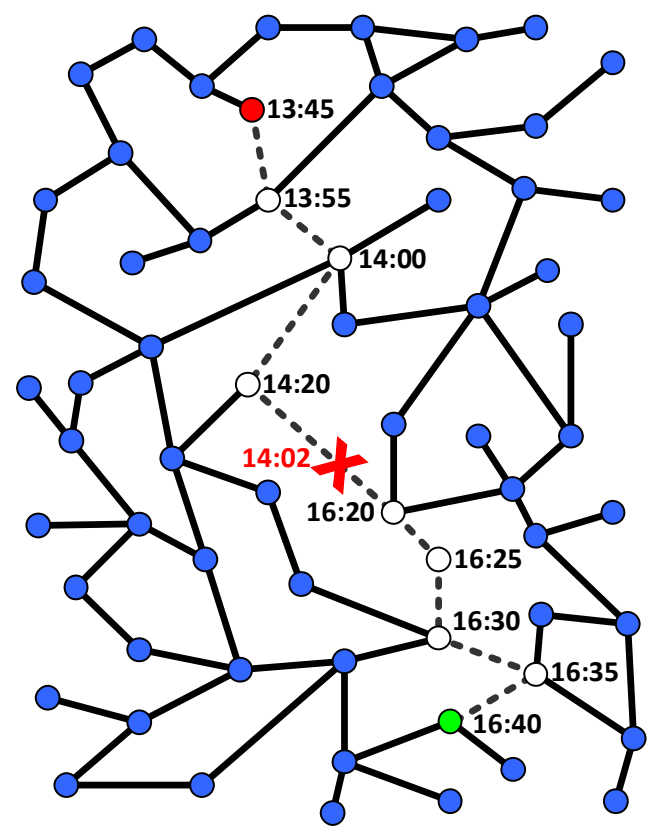

(b) Network with exceptional event

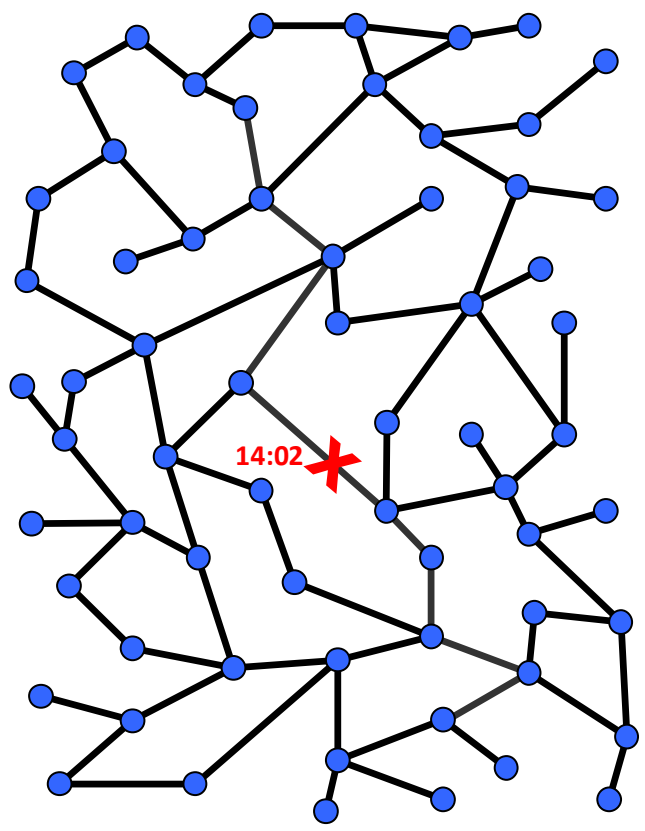

X Event that blocks a link

(d) Network with exceptional event and adapted route

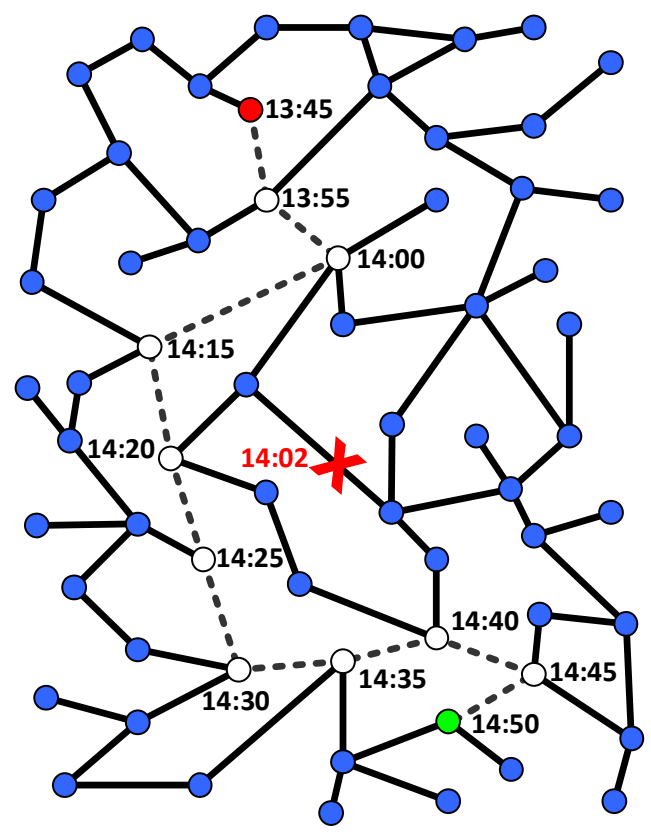


Figure 1 shows a simple example for a scenario, where an iterative approach would produce illogical and wrong results. In Figure 1(a) an agent's planned route in a sample network is shown, including the times when the driver passes each node of the route. Clearly, those times are only valid, if no exceptional event occurs. Figure 1(b) shows a link where an event like an accident happens which blocks that link for two hours. As a result, the agent reaches its destination two hours later than expected (Figure 1(c)). When this scenario is iterated, the agent recognizes that its route has a much higher travel time than expected and therefore it will choose another route. The traffic jam caused by the accident will probably also increase the travel times on links next to the blocked link. Therefore, the agent might find a route which is quite different than the original one (Figure $1(\mathrm{~d})$ ). A closer look at the node where the new route differs for the first time from the original one shows, that this happens even before the accident had happened, which is here not feasible and illogical.

An obvious solution to avoid problems like this is using an alternative simulation approach without an iterative optimization process. The next section discusses such an approach and the requirements that is has to fulfill.

\subsection{Within-Day Replanning Approach}

A within-day replanning approach uses a strategy that differs significantly from the one used by an iterative approach. Instead of multiple iterations only a single one is simulated. Thus, it is necessary that the agents can adapt their plans during this iteration without having information from previous iterations available. To do so, they have to continuously collect information and take into account their desires, beliefs and expectations when they decide how to (re)act.

While iterative approaches can use best-response modules, a within-day approach has to use something one might call a best-guess module. The travel times are an obvious example. In an iterative approach, the travel times can be collected from the previous iteration or even be averaged over several past iterations. The nearer a stable system is to a relaxed state, the smaller are the differences in the travel times between two iterations. This is not possible in a within-day approach. Even if an agent has perfect knowledge, it can only assume how the traffic flows will evolve in the future. To do so, it can take different information into account to estimate the travel times. It could for example take travel times from a typical day without exceptional events and combine them with information it gathers during the simulated day. Depending on the amount and the quality of this information, the agent might rely more or less on its experience.

Therefore, the decision making process of an agent becomes an important topic. In an iterative approach, each agent has total information and can thus select the best route. Due to the limitation of available information this is not possible in a within-day approach. One agent could 
for example choose a route where the expected travel time is very short but also very uncertain. Another agent might not be willing to take that risk and therefore select a longer route where the travel time is more reliable. Also the perception of information might vary between agents. One will probably rely on traffic information from the media, another one might ignore it.

Each within-day replanning action is categorized by two parameters - the replanned element of the plan (an activity or a trip) and the point in time when the replanned plan element is executed (right now or at a future point in time). If an activity is replanned, several changes are possible. Its start and end time can be adapted, its location can be changed, it can be dropped or created new from scratch. For a trip the origin and the destination, the route, the mode of transport and the departure time can be replanned. Often replanning one plan element results in a chain reaction that forces other plan elements also to be replanned. If for example an activity is dropped, the trips from and to this activity have to be merged.

The second parameter that categorizes a replanning action depends on the point in time when the replanned plan element is executed. This could be either the currently performed plan element or one that is planned to be performed in the future. It is obvious, that in a currently performed plan element not all previously mentioned replanning actions can be conducted. E.g. the start time of an activity or the transport mode of a trip that is currently performed cannot be adapted anymore.

Due to the limited available information, a within-day replanning approach will, in contrast to an iterative approach, not converge to a user equilibrium. Decisions made during the simulated time period may seem to be optimal when they are made. However, if they are evaluated retrospectively, an agent might realize that they were not.

\subsection{Combined Approaches}

Besides iterative or within-day replanning only approaches, it is also possible to combine them. An obvious application is solving situations that cannot be planned exactly in advance like parking or car-sharing. An agent is for example able to plan a parking activity, but it cannot be foreseen which parking lots are available when the agent arrives. Thus, within-day replanning can be used when the agent starts its parking choice. Other agents might want to share their car. To do so, it has to be ensured, that they really meet. This can be ensured using within-day replanning. If the driver arrives to early, a waiting activity is added to its plan, otherwise the picked up agent will perform a waiting activity until the car arrives. 


\section{Behavior Modeling}

Typically, behavioral models are used in the field of transport planning assume, that each person tries to improve its daily schedule and the joint optimization of all people leads to a user equilibrium. However, depending on the kind of exceptional event, such a behavioral model is not appropriate anymore. An example where such a model still might be usable is a scenario where an accident causes a small-scale traffic jam. The affected people might be only induced to adapt their route to still reach their destinations in time. However, for large-scale exceptional events more complex behavioral models are required.

One might think of a large flooding that results in the evacuation of the affected region. There, a person will evaluate different options of how to react. A first option would be trying to reach a safe area immediately. Another one would be to first try to meet some family members first and then leaving the affected area together. To allow a person to choose between such alternatives, a detailed and flexible behavioral model is required. A common implementation of such a model is the so called BDI (beliefs, desires, intentions) approach (e.g. Wooldridge, 2000; Eymann, 2003).

In the BDI approach, the level of information of an agent is represented by its beliefs, e.g. about the behavior of other agents or the traffic flows in a certain region. As the term belief indicates, some information might be wrong or misinterpreted and, therefore, some beliefs may not necessarily be true. If an agent receives new information, it will update its beliefs. Some beliefs might be based on facts (e.g. the person saw the flooded area), others are based on assumptions (the area is flooded, therefore also the desired evacuation route will not be passable anymore) or hearsay.

What people are trying to reach is described by their desires. Typically, a person has multiple desires that often compete with each other (e.g. evacuate directly versus meet family to evacuate together). Therefore, the people have to weight their desires because trying to reach all of them might result in reaching not even one. Finally, the intentions of a person describe what the person plans to do. In general, the beliefs, desires and intentions of a person interact and change over time. A person might get new information which leads to updated beliefs, changed weight of a desire and therefore result in changed intentions.

When implementing the BDI approach in an agent-based simulation, information on sociodemographics as well as a model that describes the beliefs, desires and intentions of the agents are needed. Moreover, a model that describes the information flows-which agents get which information at what time-and their perception is required.

Each type of exceptional events has unique characteristics. Following the BDI approach this means, that a behavioral model for each type of exceptional event needs to be formulated. 
Currently, a simple behavioral model, that will be refined using the results of the literature review described above, is used.

\section{MATSim Framework}

MATSim (Multi Agent Transport Simulation) is a framework for iterative, agent-based microsimulations of transport systems. It is currently developed by teams at ETH Zurich and TU Berlin as well as the senozon AG, a spin-off company founded by former members of both institutes. Balmer (2007) gives a detailed description of the framework, its capabilities and its structure. Because of its agent-based approach, every person in the system is modeled as an individual agent in the simulated scenario. Each agent has personalized parameters such as age, sex, available transport modes and scheduled activities. Due to the modular structure of the simulation framework, an agent's parameter set can be easily extended by new attributes, for example personalized preferences and desires. The application of MATSim to a large scale scenario of Switzerland (over 6 million agents simulated on a high resolution network with 1 million links) is presented by Meister et al. (2010).

The framework supports time variant networks, which is an important feature in the context of exceptional events that influence the network infrastructure. Changes in the available capacity as well as the speed limit are supported. For example, one might think of a traffic accident that blocks one of multiple lanes. On the one hand, this would clearly reduce the capacity of the road, on the other hand the police might additionally reduce the speed limit around the scene of the accident.

Figure 2(a) shows the structure of a typical, iterative MATSim simulation run. After the creation of the initial demand, the plans of the agents are modified and optimized in an iterative process until a relaxed state of the system (typically a user equilibrium) is found. The analysis of the results can be performed afterwards.

The loop shown in the figure contains the elements execution (simulation), scoring and replanning. Within the simulation module, the plans of the agents are executed. Afterwards, the scoring module uses a utility function to calculate the quality of the executed plans. The basic utility function for MATSim is described by Charypar and Nagel (2005). Based on the results of the scoring module, the replanning module creates new plans by varying start times and durations of activities as well as the routes and modes used to travel from one activity to another. Replanning modules currently under development will additionally allow to change order of the planned activities (Feil, 2010) as well as the locations where they are performed (Horni et al. 2009). 
Figure 2: Structure of the MATSim loop

(a) Iterative MATSim loop

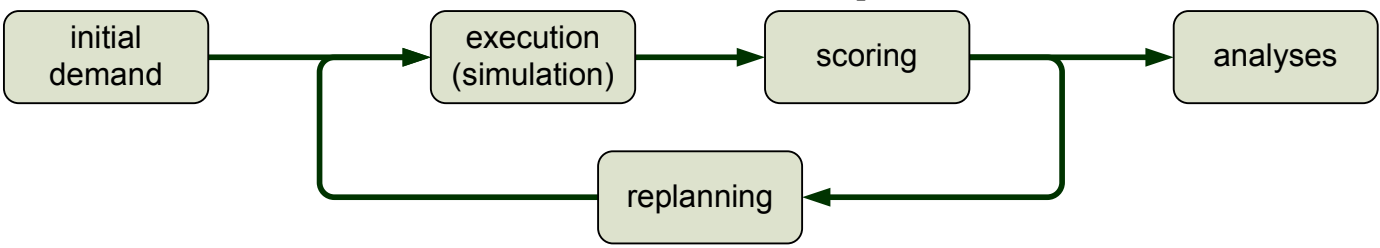

(b) (Iterative) within-day replanning MATSim loop

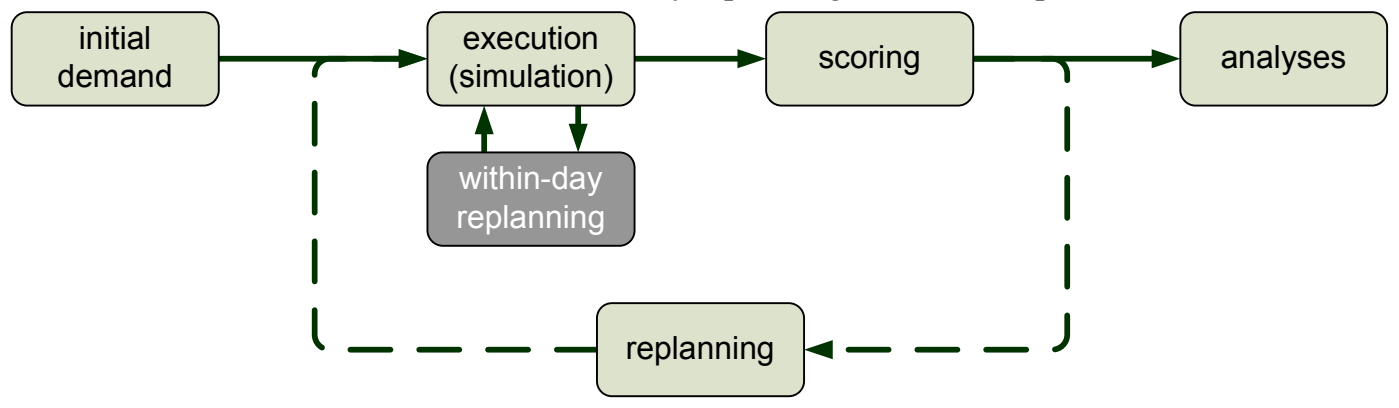

By default, all agents try to optimize their plans using the same objective function. However, it is possible to respect agent specific parameters. Due to the modular structure of the simulation framework, a more complex behavioral model, like the previously described BDI approach, can easily be implemented.

When adding within-day replanning to MATSim, the iterative loop has to be adapted as shown in Figure 2(b). On the one hand, the additional within-day replanning module is added, which iteracts with the mobility simulation. On the other hand, doing more than one iteration is only necessary, if a combined simulation approach is used.

Simulation of the traffic behavior is also part of the iterative loop. The task of the simulation module is to execute the plans of the agents within the simulated scenario. The so called QSim is a deterministic, Java based implementation of a queue model and uses a time step based approach with a step size of one second. Within each time step, the state of the queues is considered. By default, only car traffic is simulated physically. The travel times for other modes like walk, bike or public transport are estimated based on the crow fly distance. However, support for public transport has been added recently (Rieser, 2010).

\section{Implementation}

In order to simulate within-day replanning, the agents have to continuously collect information. Each time they get new information, they have to check whether they want to adapt their 
Figure 3: Implementation into the QSim

(a) QSim time step

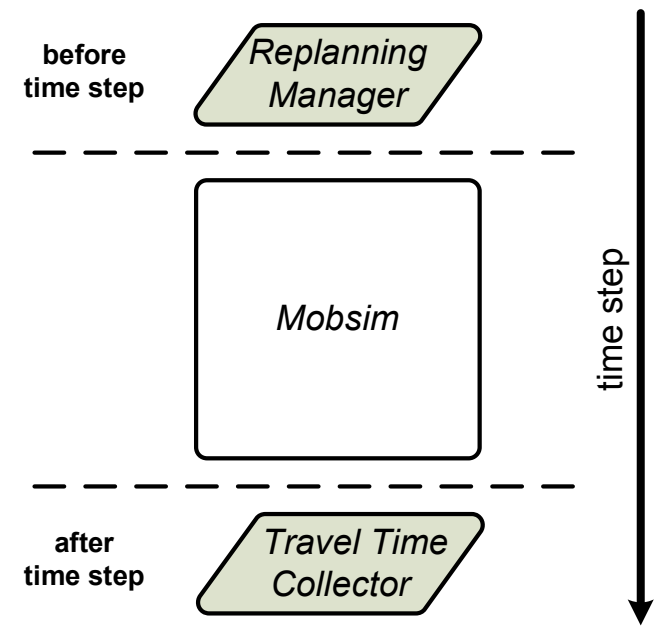

New Module

\section{Existing Module}

(b) Replanning Manager

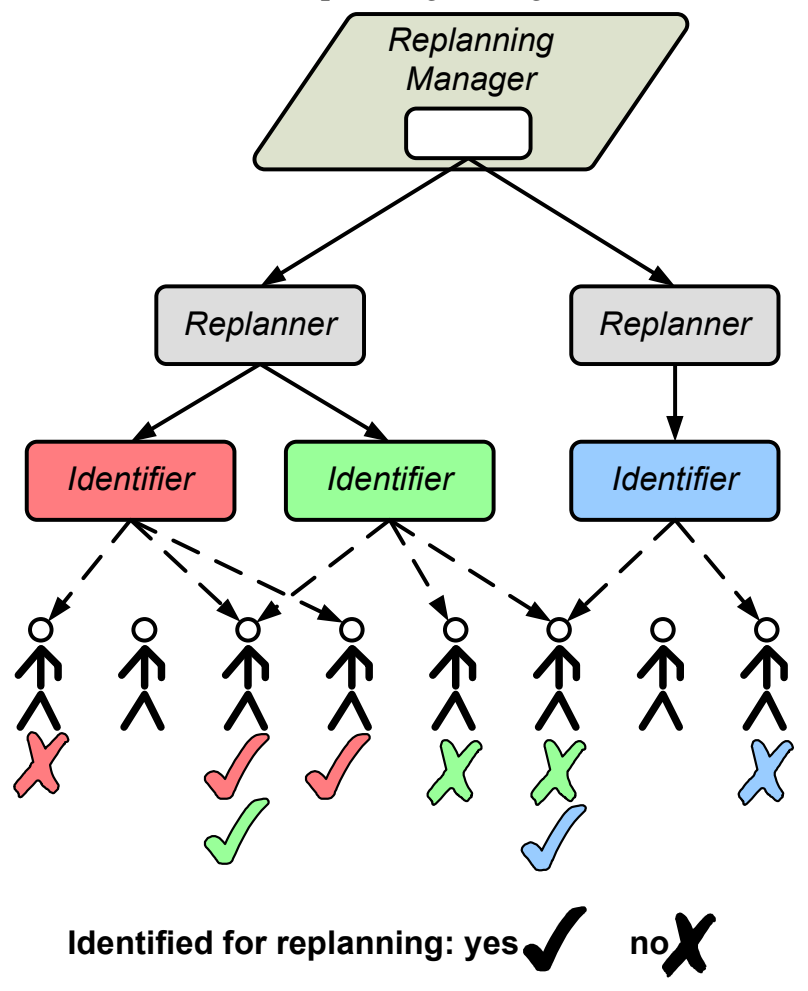

scheduled daily routine. In a time step based approach, as it is implemented in the QSim, new information can be distributed to the agents once in every time step. Figure 3(a) shows the structure of a time step. By default, only the Mobsim (mobility simulation) is executed, which simulates the movement of the agents. However, it is possible to perform additional operations before the time step starts and after it has ended. In the figure, two modules, which are part of the within-day replanning framework, have been added.

The first one is called Replanning Manager. Its task it to control the entire within-day replanning process. A conceptional overview is given in Figure 3(b). Multiple Replanners can be registered to the Replanning Manager. Each Replanner represents a unique replanning strategy like re-routing or time mutation and uses a set of Identifiers. The Identifiers communicate with the agents and selects those agents, who are given the opportunity to adapt their plans. An Identifier can be seen as an information distributing unit like a radio station or a policeman. Therefore, not every Identifier communicates with all agents. E.g. agents that are at home will probably listen to the radio but agents walking in the park will not. Each Identifier returns a list of agents to its superior Replanner, which then adapts the plans of those agents. Afterwards the Mobsim simulates the current time step.

For every possible kind of replanning, a Replanners is available. Trips as well as activities can 
be adapted. Clearly, some replanning operations are not available anymore, if a trip or activity has already been started. The possible adaptations are:

- current trip (route, destination)

- future trip (add, remove, mode, route, origin, destination)

- current activity (end time)

- future activity (add, remove, location, type, start and end time)

For complex plan adaptations those basic Replanners can be combined. If for example an agent which is currently performing a trip changes the destination of its next activity, additionally the routes of the current and the next trip have to be adapted.

Additionally, four basic Identifiers have been implemented so far. They identify agents, which are:

- performing an activity

- performing an activity which will end in the current time step

- performing a trip

- performing a trip and are going to move to another link

More complex Identifiers, which will also include a behavior model, will be part of the future work on the framework.

The so called Travel Time Collector is a new module that is executed before each time step ends. Its task is to provide actual link travel times to the Replanners. This is done by collecting and averaging the travel times of agents that have recently passed a link during a given time span. A typical time span is 15 minutes, older link travel times are ignored. The duration of the respected time span has an important impact on the travel times that are reported to the Replanners. On the one hand, significant changes in the link travel times will be communicated very slowly, if the time span is too long. On the other hand, a too short duration will result in overrating small changes.

The Travel Time Collector is a simple, but efficient implementation of a within-day travel time calculator. On the one hand, it does not implement features like traffic flow predictions or dynamic weighting of recent travel times based on historical data. On the other hand, due to the abandonment of such features, it is very robust, even in scenarios where the traffic flow conditions change dramatically.

Performing the replanning in each time-step before the Mobsim is executed, is very convenient. On the one hand, the Mobsim is paused and, as a result, all simulated objects (i.e. agents, links and facilities) are in a fixed condition. On the other hand, it is very simple to use multiple parallel threads to perform the replanning. This results in an almost linear speed-up. 
For the experiments that are described subsequently, a simple behavioral model is applied, where the only replanning operation that is available for the agents is replanning the route of a currently performed trip. Experiments with more replanning alternatives, like location choice or activity chain order, will be part of the future work.

In the behavioral model, the beliefs of the agents are based on information they obtain from the Travel Time Collector. They all try to minimize their travel times, which in turn maximizes the time they can spend performing activities. The desires and intentions of an agent are predefined by its initial plan. Since the agents are only allowed to adapt their routes, their desires will not change during the simulation. However, based on the information from the Travel Time Collector, the agents can adapt their intentions to satisfy their desires.

\section{Scenario}

To validate the capabilities of the proposed within-day replanning framework, it is applied to a model of the Canton Zurich. Several runs using different sets of parameters for the within-day modules are conducted. The scenario contains 67.000 agents, which is a $10 \%$ sample of the relevant population, and uses a planning network with 24.000 nodes and 60.000 links. The simulation area and the traffic network are shown in Figure 4(a). The thick black line marks the Canton Zurich. Figure 4(b) depicts the distribution of the performed trips over the simulated period.

It is assumed, that the capacity of several arterial roads (red links in Figure 4(c)) in the center of the city of Zurich is reduced to $20 \%$ of their initial capacity . The incidents are modeled using MATSim's time variant networks. At 07:00 a.m. the capacities are reduced, at 09:00 a.m. they are reset to their original values. Figure 4(d) shows the distribution of the conducted trips, when the event occurs but the agents are not allowed to adapt their routes. A comparison of the trip distribution with and without the event is shown in Figure 4(f) As the figure shows, the system needs until 16:00 to normalize.

Within-day replanning is enabled when the incidents occur and disabled three hours after the capacities have been set back to their original values. This additional hours give the agents the opportunity to take the fact into account, that the capacities have been reset. It is further assumed, that only agents that would travel over the affected links in the time window where the link capacities are reduced, will use within-day replanning. Moreover, those agents will only react by adapting their routes if they are within a certain distance to the affected links $(0.0 \mathrm{~km}$, $1.0 \mathrm{~km}, 2.5 \mathrm{~km}$ and $5.0 \mathrm{~km}$; the areas are shown in Figure $4(\mathrm{e}))$. Other replanning operations than rerouting, e.g. relocating an activity to another location, are not enabled. Additionally, the share of agents that use within-day replanning is varied $(0 \%, 25 \%, 50 \%, 75 \%$ and $100 \%)$. 
Figure 4: Scenario without replanning

(a) Simulation area

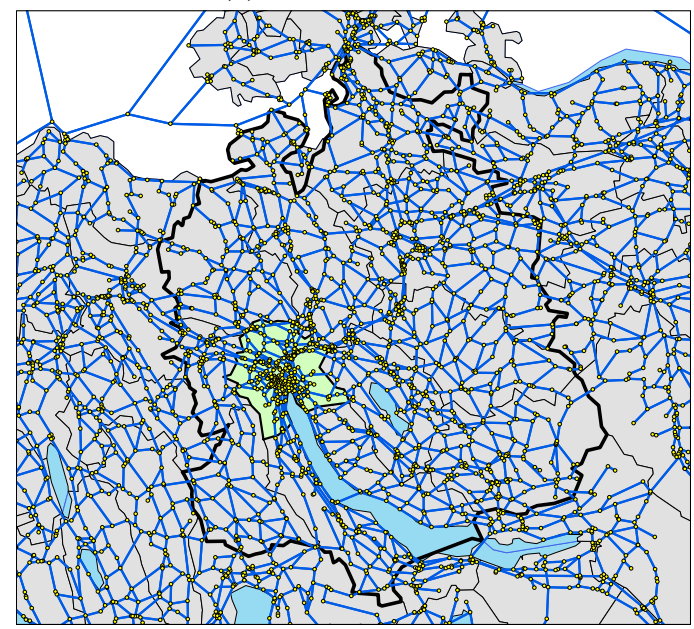

(c) Links with reduced capacities

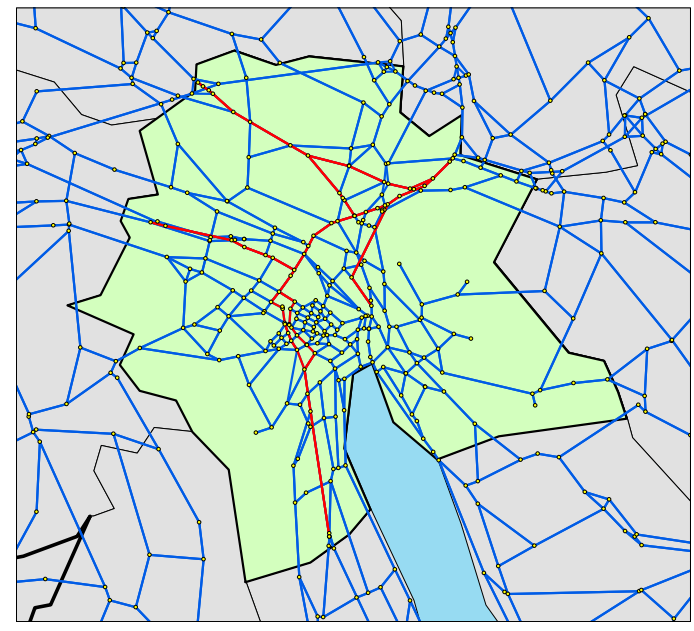

(e) Distance to affected links

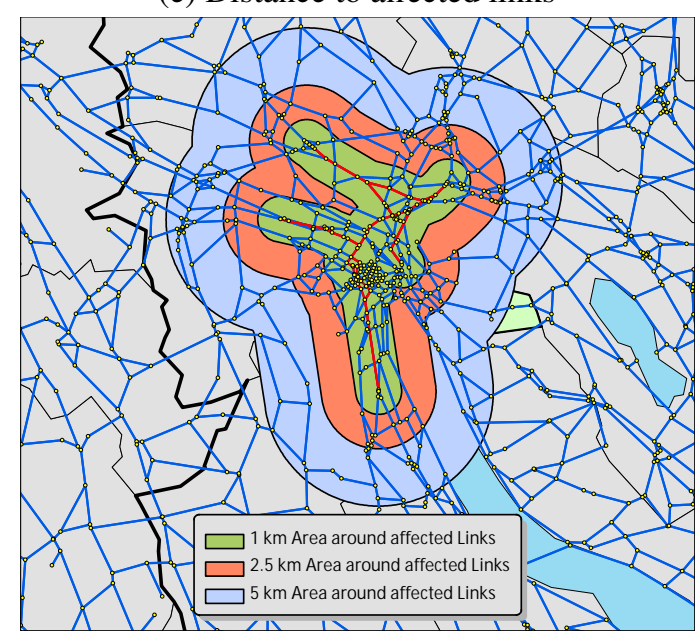

(b) Trip histogram in a relaxed state

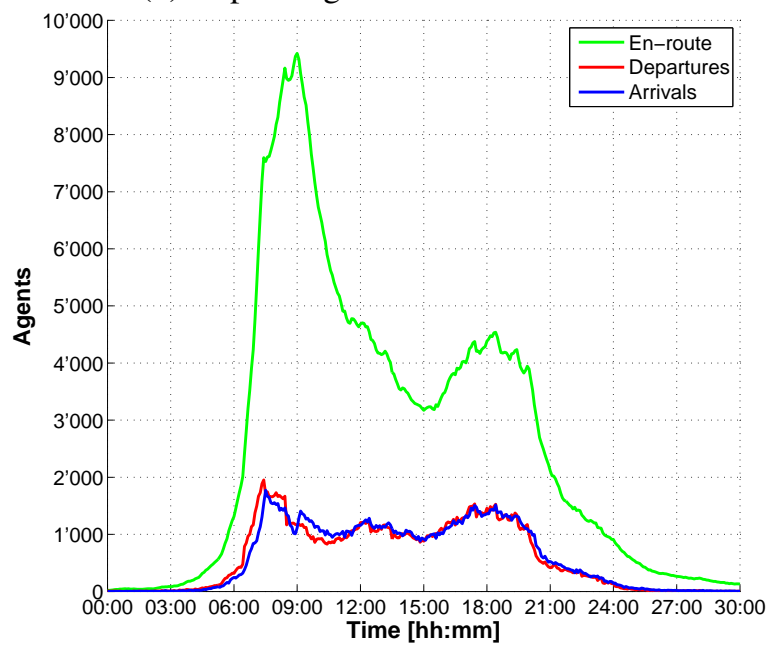

(d) Trip histogram with event

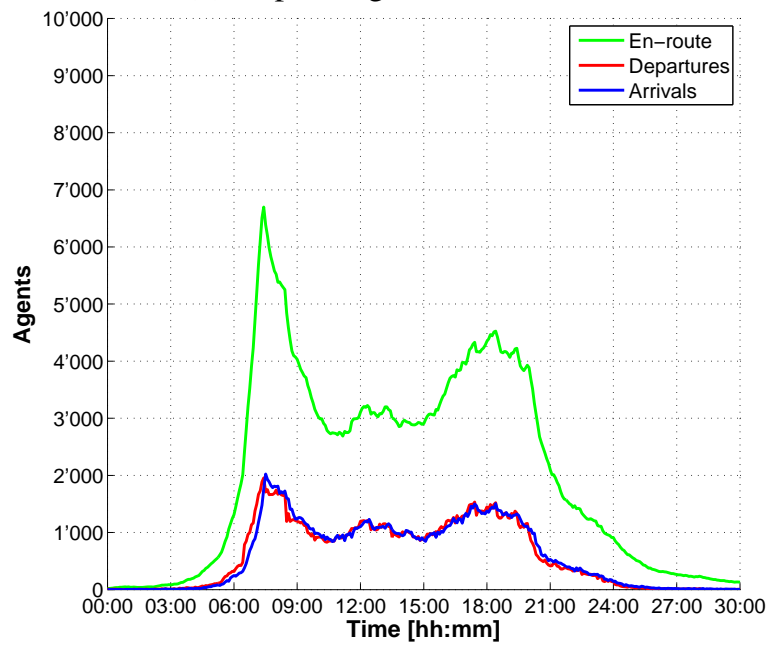

(f) Agents en-route with and without event

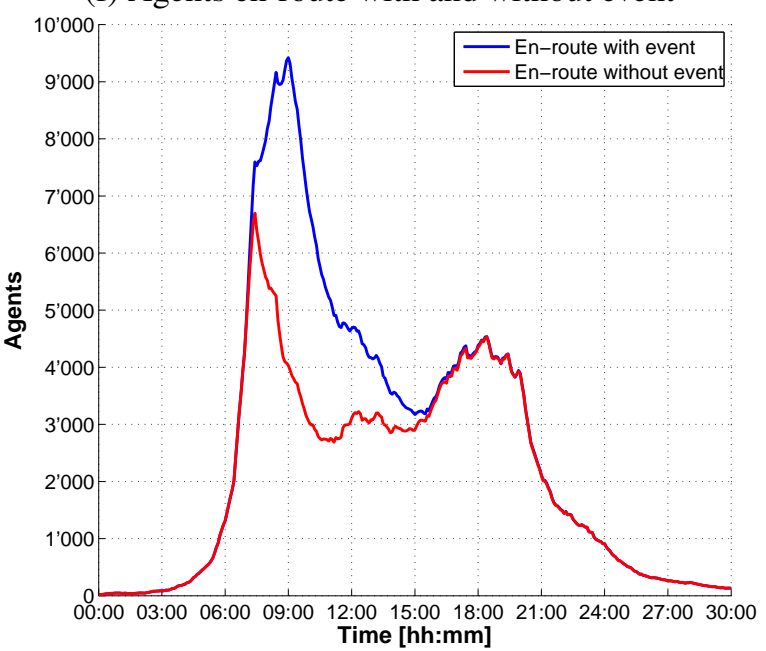




\section{Results}

The results of the experiments are shown in Figure 5. The effect of different replanning shares is shown in Figures 5(a) to 5(d), Each figure depicts a different replanning buffer, which defines the area wherein the agents can adapt their plans. In Figure 5(a), the agents can only replan their routes while they are located on one of the affected links. The results show, that there is still a lot of room for improvement. This improvement is realized with higher replanning buffer as shown in Figures 5(b) to 5(d). However, among themselves, the results differ only imperceptibly.

As one can expect, the more agents use within-day replanning, the faster the traffic flows normalize, but the improvement between $75 \%$ and $100 \%$ replanning share is negligible.

The mean travel times of those agent who use within-day replanning are shown in Figures 5(e) and 5(f). Again it is shown that the agents are able to find better routes, if they start replanning before they have entered one of the affected links and that increasing the replanning buffer over a certain value will not further reduce the mean travel times. While there is a large improvement from $0.0 \mathrm{~km}$ to $1.0 \mathrm{~km}$, the difference between the $1.0 \mathrm{~km}$ buffer and the $2.5 \mathrm{~km}$ buffer is already much smaller. Comparing the $2.5 \mathrm{~km}$ and the $5.0 \mathrm{~km}$ buffer shows, that the mean travel times are almost equal.

Another finding from Figures $5(\mathrm{e})$ and $5(\mathrm{f})$ is that the mean travel times can increase again, if too many agents use within-day replanning. This might be a result of the limited amount of available information. An agent chooses a new route using estimated travel times from the Travel Time Collector which calculates the travel times based on data collected over the past 15 minutes. To overcome this phenomenon, the Travel Time Collector could be further improved, e.g. by adding a logic that tries to predict future travel times based on the trend of the data in the past.

\section{Conclusion and Outlook}

In this paper, we have introduced a within-day replanning approach as well as its fields of applications. We have also shown, why traditional approaches will fail in scenarios with unexpected exceptional events while within-day replanning does not.

The evaluation of the experiments conducted shows that the implementation of the withinday replanning technique produces results as one could have expected in the sample scenario. The implemented behavioral model as well as the replanning modules provide mainly basic capabilities but due to the modular structure of the implementation, more complex models and modules can be easily added. 
Figure 5: Results

(a) Trip histogram, $0.0 \mathrm{~km}$ replanning buffer

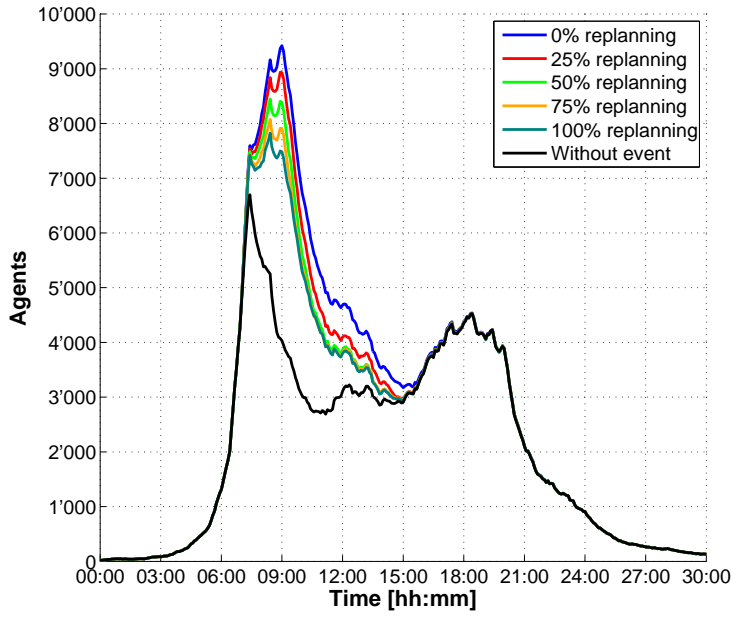

(c) Trip histogram, $2.5 \mathrm{~km}$ replanning buffer

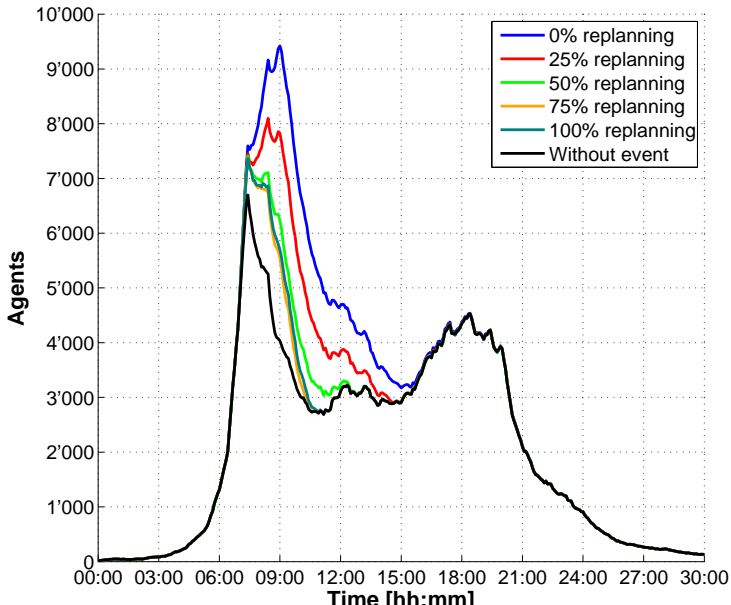

(e) Mean travel times

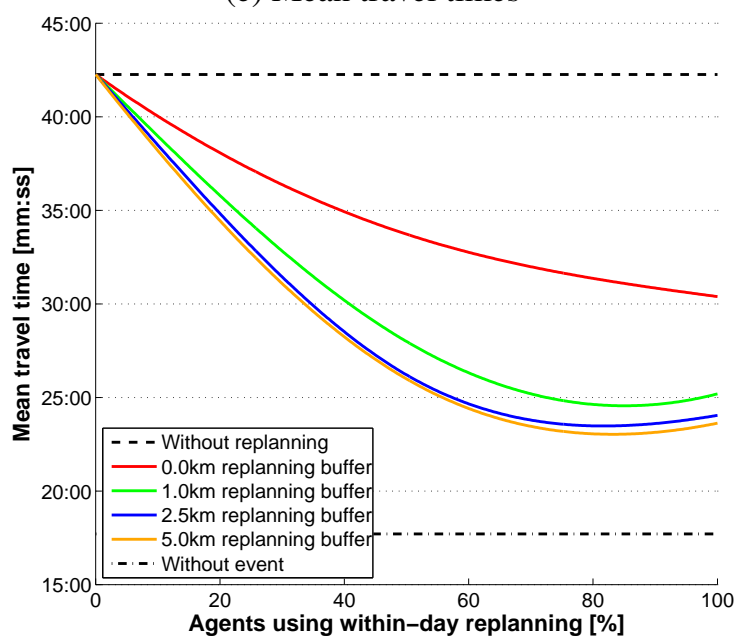

(b) Trip histogram, $1.0 \mathrm{~km}$ replanning buffer

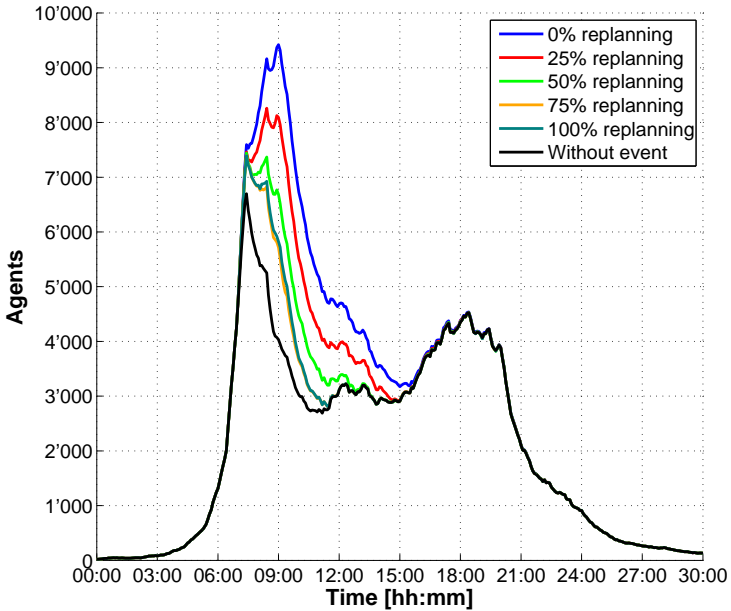

(d) Trip histogram, 5.0km replanning buffer

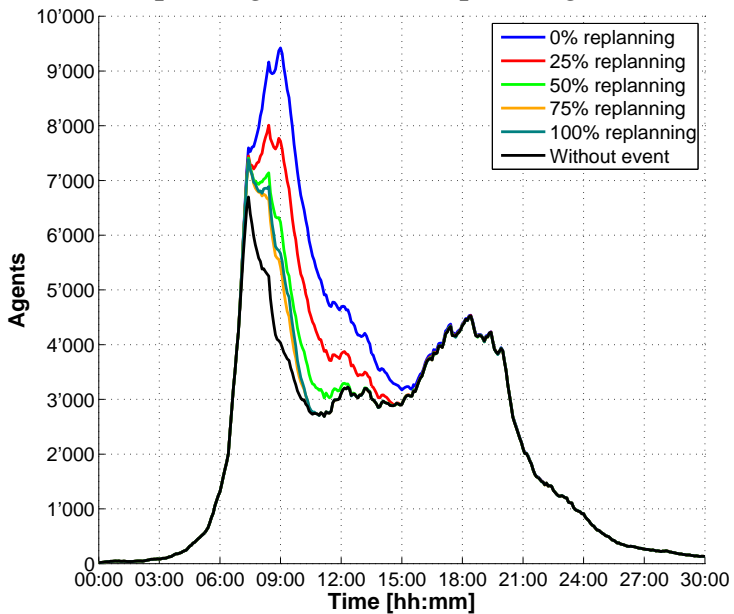

(f) Mean travel times 3d

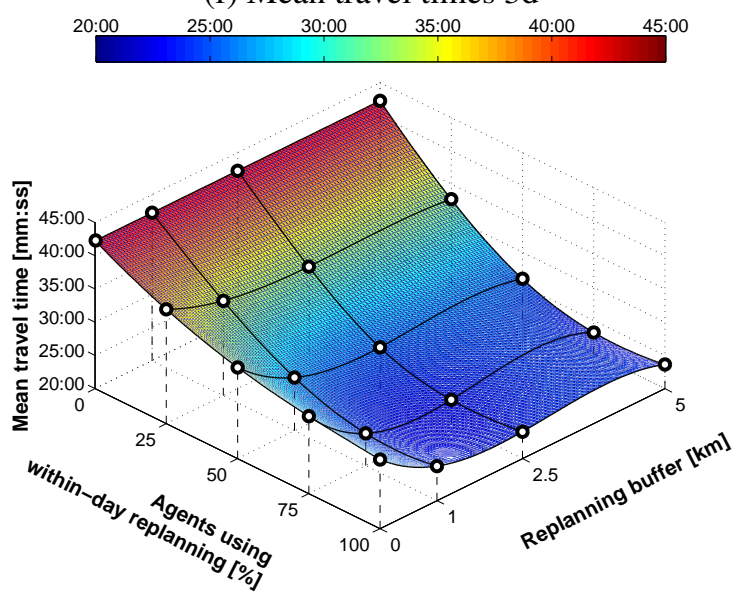


Future steps will be the full integration of the BDI approach into the framework. Based on this and the findings from the literature review in the field of behavior under evacuation conditions, a flexible behavioral model for extreme situations like natural hazards will be implemented and applied to sample scenarios. Doing so will hopefully allow us to model such scenarios with a much higher level of detail than other commonly used approaches based on the four step process or iterative agent-based simulations.

\section{References}

Balakrishna, R., Y. Wen, M. E. Ben-Akiva and C. Antoniou (2008) Simulation-based framework for transportation network management in emergencies, Transportation Research Record, 2041, 80-88.

Balmer, M. (2007) Travel demand modeling for multi-agent traffic simulations: Algorithms and systems, Ph.D. Thesis, ETH Zurich, Zurich, May 2007.

Ben-Akiva, M. E., M. Bierlaire, H. Koutsopoulos and R. Mishalani (1998) DynaMIT: A simulation-based system for traffic prediction, paper presented at the DACCORS Short Term Forecasting Workshop.

Cameron, G. D. B. and G. I. D. Duncan (1996) PARAMICS-parallel microscopic simulation of road traffic, Journal of Supercomputing, 10 (1) 25-53.

Charypar, D. and K. Nagel (2005) Generating complete all-day activity plans with genetic algorithms, Transportation, 32 (4) 369-397.

Cova, T. J. and J. P. Johnson (2003) A network flow model for lane-based evacuation routing, Transportation Research Part A: Policy and Practice, 37, 579-604.

Drabek, T. E. (1969) Social processes in disaster: Family evacuation, Social Problems, 16 (3) 336-349.

Efrat, E. (1992) The geography of a population mass-escape from the Tel Aviv area during the gulf war, The Geographical Journal, 158 (2) 199-206.

Eymann, T. (2003) Digitale Geschäftsagenten - Softwareagenten im Einsatz, Springer, Berlin.

Feil, M. (2010) Choosing the daily schedule: Expanding activity-based travel demand modelling, Ph.D. Thesis, ETH Zurich, Zurich.

Frey, B. S., D. A. Savage and B. Torgler (2011) Behavior under extreme conditions: The titanic disaster, Journal of Economic Perspectives, 25 (1) 209-222. 
Fritz, C. E. and E. S. Marks (1954) The NORC studies of human behavior in disaster, Journal of Social Issues, 10 (3) 26-41.

Fussell, E. (2006) Leaving New Orleans: Social stratification, networks, and hurricane evacuation, webpage, June 2006, http://understandingkatrina.ssrc . org/Fussell/.

Hobeika, A. G. and C. Kim (1998) Comparison of traffic assignment in evacuation modeling, IEEE Transactions on Engineering Management, 45 (2) 192-198.

Horni, A., D. M. Scott, M. Balmer and K. W. Axhausen (2009) Location choice modeling for shopping and leisure activities with MATSim: Combining micro-simulation and time geography, Transportation Research Record, 2135, 87-95.

Houts, P. S., P. D. Cleary and T. W. Hu (1988) The Three Mile Island Crisis: Psychological, Social, and Economic Impacts on the Surrounding Population, Pennsylvania State University Press, Harrisburg.

Houts, P. S., M. K. Lindell, T. W. Hu, P. D. Cleary, G. Tokuhata and C. B. Flynn (1984) The protective action decision model applied to evacuation during the Three Mile Island crisis, International Journal of Mass Emergencies and Disasters, 2 (1) 27-40.

Jha, M., K. Moore and B. Pashaie (2004) Emergency evacuation planning with microscopic traffic simulation, Transportation Research Record, 1886, 40-48.

Johnson, J. H. and D. J. Zeigler (1986) Modelling evacuation behavior during the Three Mile Island reactor crisis, Socio-Economic Planning Sciences, 20 (3) 165-171.

Kiefer, J. J., P. Jenkins and S. Laska (2009) City-assisted evacuation plan participant survey report, Research Report, New Orleans Office of Emergency Preparedness, University of New Orleans, Center for Hazards Assessment, Response \& Technology, New Orleans, April 2009.

Kowald, M., C. Dobler and K. W. Axhausen (2011) Der Einfluss sozialer Kontakte in grossräumigen Evakuationsereignissen, Working Paper, 683, IVT, ETH Zurich, Zurich.

Liverman, D. M. and J. P. Wilson (1981) The Mississauga train derailment and evacuation, Canadian Geographer, 25 (4) 365-375.

Meister, K., M. Balmer, F. Ciari, A. Horni, M. Rieser, R. A. Waraich and K. W. Axhausen (2010) Large-scale agent-based travel demand optimization applied to Switzerland, including mode choice, paper presented at the 12th World Conference on Transportation Research, Lisbon, July 2010.

Perry, R. W. (1985) Comprehensive emergency management: evacuating threatened populations, JAI Press, Greenwich. 
Quarantelli, E. L. (1980) Evacuation behavior and problems: findings and implications from the research literature, Disaster Research Center, Ohio State University, Columbus.

Quarantelli, E. L. (1990) The warning process and evacuation behavior: The research evidence, Preliminary Paper, 148, University of Delaware, Delaware.

Rieser, M. (2010) Adding transit to an agent-based transportation simulation, Ph.D. Thesis, Technical University Berlin, Berlin.

Rosengren, K. E., P. Arvidson and D. Sturesson (1975) The barsebäck 'panic': A radio programme as a negative summary event, Acta Sociologica, 18 (4) 303-321.

Schwerdtfeger, T. (1984) DYNEMO: A model for the simulation of traffic flow in motorway networks, in J. Volmuller and R. Hamerslag (eds.) Proceedings of the Ninth International Symposium on Transportation and Traffic Theory, chap. 4, 65-87, VNU Science Press, Utrecht.

Sheffi, Y., H. S. Mahmassani and W. B. Powell (1982) A transportation network evacuation model, Transportation Research Part A: Policy and Practice, 16 (4) 209-218.

Shenhar, G., D. Gidron and K. Peleg (2008) Mass population displacement under an unclear evacuation policy during the Israel-Lebanon War 2006, Journal of Homeland Security and Emergency Management, 5 (1) 1-11.

Smith, L., R. J. Beckman, D. Anson, K. Nagel and M. E. Williams (1995) TRANSIMS: TRansportation ANalysis and SIMulation System, paper presented at the 5th TRB National Transportation Planning Methods Applications Conference, Seattle, April 1995.

Stern, E. and Z. Sinuany-Stern (1989) A behavioural-based simulation model for urban evacuation, Papers in Regional Science, 66 (1) 87-103.

Wooldridge, M. (2000) Reasoning about Rational Agents, MIT Press, Cambridge.

Zeigler, D. J., S. D. Brunn and J. H. Johnson (1965) Evacuation from a nuclear technological disaster, The Geographical Review, 71 (1) 1-16.

Zelinsky, W. and L. A. Kosiński (1991) The Emergency Evacuation of Cities: A Cross-national Historical and Geographical Study, Rowman \& Littlefield, Maryland. 